\title{
0193 THE REQUIREMENTS AND CHALLENGES IN PREVENTING OF ROAD TRAFFIC INJURY IN IRAN: A QUALITATIVE STUDY
}

D K Zavareh, ${ }^{*}$ M Bigdeli, R Mohammadi, H R Khaneh, L Laflamme, A Bikmoradi, B J A Haglund Correspondence: Urmia University of Medical Sciences, Nosrat Ave, Oroumiyeh, Iran

\subsection{6/ip.2010.029215.193}

Background Road traffic injuries (RTIs) are a major public health problem, especially in low- and middle-income countries. Iran has one of the highest mortalities from RTIs Stakeholders. The aim of this study was to explore barriers to the prevention of RTIs and provide appropriate suggestions for prevention, based on the perceptions of stakeholders, victims and road-users as regards RTIs.

Method Thirty-eight semistructured interviews were conducted with informants in the field of RTI prevention. A qualitative approach using grounded theory method was employed to analyze the material gathered.

Results The core variable was identified as The lack of a system approach to road-user safety. The following barriers in relation to RTI prevention were identified as: human factors, transportation system and organisational coordination. Suggestions for improvement included education (for the general public and targeted group training), more effective legislation, more rigorous law enforcement, improved engineering in road infrastructure, and an integrated organisation to supervise and coordinate preventive activities.

Conclusion The major barriers identified in this study were human factors and efforts to change human behaviour were suggested by means of public education campaigns and stricter law enforcement. However, the lack of a system approach to RTI prevention was also an important concern. There is an urgent need for both an integrated system to coordinate RTI activities and prevention and a major change in stakeholders 


\section{IP Safety 2010 abstracts}

attitudes towards RTI prevention. The focus of all activities should take place on road users safety. 